\title{
Correction
}

\section{Correction: Pilz, Carta et al., "Functional Imaging of Dentate Granule Cells in the Adult Mouse Hippocampus"}

In the article "Functional Imaging of Dentate Granule Cells in the Adult Mouse Hippocampus" by Gregor-Alexander Pilz, Stefano Carta, Andreas Stäuble, Asll Ayaz, Sebastian Jessberger, and Fritjof Helmchen, which appeared on pages 7407-7414 of the July 13, 2016 issue, the fourth author first name was misspelled. The corrected author line is: Gregor-Alexander Pilz, Stefano Carta, Andreas Stäuble, Asli Ayaz, Sebastian Jessberger, and Fritjof Helmchen which has been corrected. Also, the authors regret an error in Figure $5 A$, where in the bottom row a duplicate trace erroneously occurred. Two episodes of example traces for the cellular calcium signal and running speed had been mistakenly inserted into the $28 \mathrm{dpi}$ (days-post-injection) session from a $32 \mathrm{dpi}$ recording session. These traces have been now replaced by two correct episodes of example traces from a 28 dpi recording session. The second sessions in the middle and bottom rows of Figure $5 A$ were actually recorded on ' 32 dpi' instead of ' 33 dpi', which has also been corrected. In addition, the last episode of example traces for ' $32 \mathrm{dpi}$ ' in the bottom row of Figure $5 A$ was missing initial data points, which have now been inserted. The description of the data in the corresponding parts of the text (Fig. 5 legend, and Results) is not affected by these changes and is still correct. The quantitative analysis (Fig. 5C), and the interpretation and conclusions of the study are also not affected as the mistake was made only during the selection of example traces for illustration. The corrected Figure 5 is shown below. Both issues have been corrected in the online PDF version.
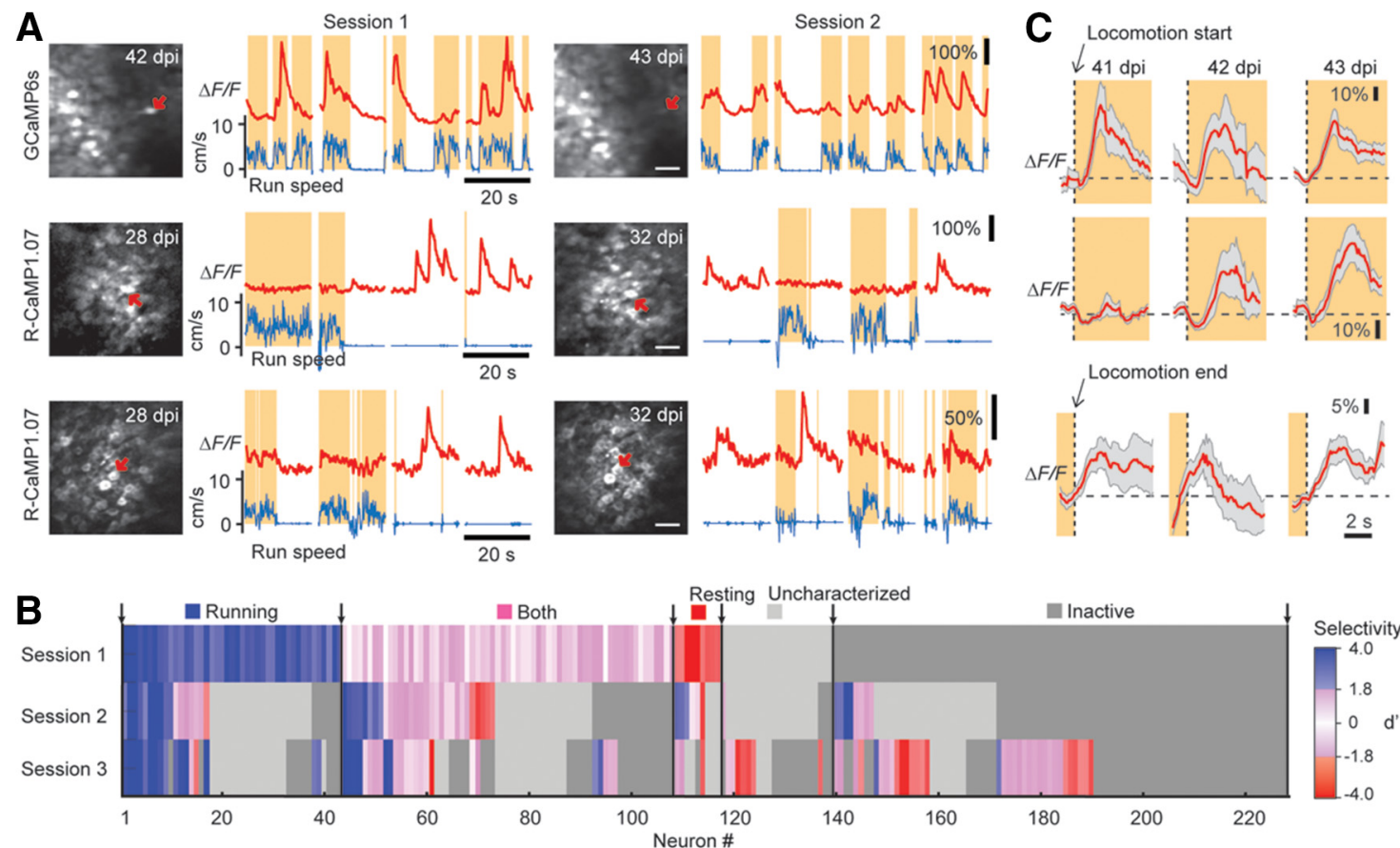

Figure 5 . 